\title{
Comunicación
}

\section{Lesiones Anatomopatológicas Producidas por Dosis Prolongadas de Fenilbutazona en una Potranca de Carrera}

\author{
Anatomopathological Lesions Produced by Prolonged Doses of \\ Phenylbutazone in a Race Horse
}

\author{
Katherine Choez A. ${ }^{1,2}$, Alfonso Chavera C. ${ }^{1}$
}

\section{ReSUMien}

El presente trabajo describe el caso de una potranca de dos años que desarrolló graves lesiones gastrointestinales después de recibir dosis prolongadas de fenilbutazona al tratar una fractura en el miembro anterior derecho. Los hallazgos de necropsia revelaron una severa gastritis catarro- hemorrágico-ulcerativa difusa aguda y edema submucoso y severa enterocolitis mucohemorrágica difusa aguda y esplenomegalia con severa congestión pasiva aguda. La causa de muerte se debió a un paro cardio-respiratorio y coagulopatía con disturbio circulatorio y proceso ulcerogénico gástrico asociado a shock iatrogénico por fenilbutazona.

Palabras clave: lesiones anatomopatológicas; fenilbutazona; equino

\section{ABSTRACT}

The present paper describes the case of a two-year-old filly who developed severe gastrointestinal lesions after receiving prolonged doses of phenylbutazone when treating a fracture in the right forelimb. Necropsy findings revealed severe acute diffuse catarrahhemorrhagic-ulcerative gastritis and submucosal edema and severe diffuse acute mucohemorrhagic enterocolitis and splenomegaly with severe acute passive congestion. The cause of death was due to cardio-respiratory arrest and coagulopathy with circulatory disturbance and gastric ulcerogenic process associated with iatrogenic shock by phenylbutazone.

Key words: pathological lesions; phenylbutazone; equine

\footnotetext{
${ }^{1}$ Laboratorio de Histología, Embriología y Patología Veterinaria, Facultad de Medicina Veterinaria, Universidad Nacional Mayor de San Marcos, Lima, Perú

2E-mail: kathyvet3@hotmail.com
}

Recibido: 6 de mayo de 2017

Aceptado para publicación: 27 de agosto de 2017 


\section{INTRODUCCIÓN}

Los caballos de carrera, debido a su alta exigencia atlética, sufren con mayor frecuencia de lesiones musculoesqueléticas. Estas lesiones producen importantes pérdidas económicas para la industria hípica, representando cerca del $82 \%$ de los problemas de pérdidas de rendimiento a causa de las cojeras (Halper et al., 2006). El tratamiento médico convencional de las cojeras incluye la utilización de antiinflamatorios no esteroideos (AINE) (Melo et al., 2009) debido a su acción analgésica, antiinflamatoria, antipirética, antitrombótica y antiendotóxica (MacAllister, 1994), además de ser medicamentos fáciles de adquirir y de costo accesible (Matthews y Carroll, 2007).

El AINE más utilizado en caballos es la fenilbutazona, ácido enólico derivado de la pirazolona (Kollias-Baker y Cox, 2004). La fenilbutazona tiene una alta afinidad a las proteínas plasmáticas (albúmina), lo que limita su distribución, pero facilita el pasaje hacia el exudado inflamatorio, originando una mayor concentración en el lugar de la lesión (Tobin et al., 1986). Después de su biotransformación hepática, da lugar a dos metabolitos: la oxifembutazona y el ã-hidroxifembutazona; siendo la primera farmacológicamente activa (Mealey et al., 1997). Estos metabolitos representan aproximadamente el $25 \%$ del fármaco total administrado, los cuales son excretados posteriormente en la orina (Lees y Higgins, 1985).

La acción farmacológica de la fenilbutazona es la inhibición no selectiva de la ciclooxigenasa $1(\mathrm{COX}-1)$ a partir de las endoperoxidasas cíclicas $\left(\mathrm{PGH}_{2}\right)$ impidiendo la formación de prostaglandinas $\left(\mathrm{PGE}_{2}\right.$, $\mathrm{PGF}_{2 \mathrm{a}}, \mathrm{PGD}_{2}$ ), prostaciclina $\left(\mathrm{PGI}_{2}\right) \mathrm{y}$ tromboxanos (TXA y TXB $_{2}$ ) (Lees Lees y Higgins, 1987). Estos compuestos eicosanoides son sintetizados por varias células y producen una gran variedad de efectos en el organismo, englobando prácticamente a todas las funciones biológicas, incluyendo en la mediación del dolor, la fiebre y la inflamación (Botting, 2006). Por ello, se cree que la inhibición de COX-1 está vinculada con los cuadros de toxicidad gastrointestinal, toxicidad renal, toxicidad hepática, hemorragias y coagulopatías, entre otros (Bricks y Silva, 2005).

Los signos iniciales de toxicidad gastrointestinal incluyen decaimiento, anorexia, depresión, diarrea, pérdida de peso y cólico ocasional (Skarra, 1994). Posteriormente se puede observar signos clásicos de shock hipovolémico por hemorragia gastrointestinal (Sumano y Ocampo, 2006b), además de hepatopatías, nefropatías, edema abdominal y edema facial (Lees y Higgins, 1985). Los factores que influyen en la presentación de cuadros de toxicidad son la formulación, la vía de administración, la duración del tratamiento, la dosis empleada, el horario de alimentación, la raza y la edad; donde algunos equinos pueden llegar a desarrollar la intoxicación con dosis terapéuticas (Jones, 2003; Melo et al., 2009).

En equinos, la dosis de fenilbutazona varía entre 2.2 y $4.4 \mathrm{mg} / \mathrm{kg} /$ día, vía endovenosa, y de 4.4-8.8 mg/kg/día, vía oral (Kollias-Baker y Cox, 2004). Su acción es de larga duración, a pesar de tener una vida media relativamente corta ( 3 a $8 \mathrm{~h}$ ) (Tobin et al., 1986; Keegan et al., 2008). Se sospecha que su larga duración es debida a la oxifembutazona y a la baja tasa de excreción por la acumulación de la fenilbutazona y oxifembutazona en el exudado inflamatorio (Lees y Higgins, 1987; Martín-Jiménez y Papich, 2002). Además, aun cuando los niveles plasmáticos de fenilbutazona han descendido, la actividad antiinflamatoria persiste debido a la compleja unión con las ciclooxigenasas (Sumano y Ocampo, 2006b). En consecuencia, los cuadros de toxicidad pueden ocurrir incluso semanas después de haber suspendido su administración, por lo que se recomienda no superar la dosis máxima del fármaco y no administrar por periodos de tiempo prolongados (Kollias-Baker y Cox, 2004). 


\section{Reporte de Caso}

\section{Anamnesis}

Se remitió el cadáver de una potranca de 2 años de edad para la necropsia. El Médico Veterinario remitente refirió que el animal murió tras culminar una artroscopia. Se indica que durante la cirugía notaron que el pulso se encontraba débil y debajo de lo normal y que durante la incisión sobre el miembro a operar no salía sangre. No se habían realizado exámenes previos para evaluar el riesgo quirúrgico, toda vez que era un animal joven y que durante sus actividades de ejercicio no había presentado problemas.

La potranca tuvo una fractura a nivel del carpo derecho ocho días atrás durante un entrenamiento físico de rutina, por lo que fue tratada con AINEs (fenilbutazona) hasta el día de la cirugía para aliviar el dolor. En el procedimiento quirúrgico se utilizó xilacina, promazil y ketamina como inducción y sevoflurano en la cirugía.

La necropsia se realizó 2-3 horas de fallecido el animal, observándose rigor mortis, mucosas levemente pálidas, condición corporal $3 / 5$ y presencia de vendaje de cirugía en miembro anterior derecho.

\section{Hallazgos de Necropsia (ver Figura 1)}

- Al exponer la piel y tejido subcutáneo de la cavidad abdominal fluía gran cantidad de sangre y linfa a modo de chorros continuos que al contacto con el exterior no coagulaba.

- El estómago distendido por gas y casi sin contenido, pero con presencia de secreción mucoide verduzca amarillenta. Pared levemente engrosada y brillosa. Mucosa muy enrojecida.

- En la «corbata suiza» se observó pérdida de solución de continuidad, de bordes irregulares. Color amarillento-verdoso a nivel de la región pilórica con varias áreas de pérdida de mucosa.
- El intestino delgado con las arterias mesentéricas congestionadas y al corte regular contenido mucoide verduzco. Mucosa con focos enrojecidos y pared brillante.

- El intestino grueso con leve contenido alimenticio verde. En la parte final del colon y recto abundante contenido de heces blandas, de coloración verde, con sustancia mucoide verduzca.

- Los linfonódulos mesentéricos en ligero aumento de tamaño y al corte enrojecidos.

- El hígado con leve aumento de tamaño, bordes redondeados y superficie lisa de coloración marrón oscura, que al corte fluía gran cantidad de sangre.

- El bazo severamente aumentado de tamaño, ocupando gran cantidad del espacio abdominal hasta la cavidad pélvica. De bordes redondeados, superficie rugosa y coloración rojiza oscuro. Al corte fluía abundante cantidad de sangre. Superficie de corte irregular con marcados puntos enrojecidos oscuros.

- Las glándulas adrenales de coloración rojiza.

- Los riñones de coloración rojiza. Al corte, la porción medular se hallaba enrojecida.

- Los ovarios y cuernos uterinos sin cambios considerables.

- Los pulmones insuflados, ruido de positividad, color rosáceo. Al corte fluía leve cantidad de sangre.

- El corazón con el ventrículo derecho contraído sin relajación muscular. Al corte se halló abundante sangre sin coagular en ambas cámaras, así como en las grandes venas y arterias.

- La mucosa del esófago estaba enrojecida.

- La tráquea sin cambios considerables.

- No se expuso la bóveda craneana.

\section{Diagnósticos Anatomopatológicos}

Esplenomegalia con severa congestión pasiva aguda, leve hepatomegalia congestiva y cambio graso, severa gastritis catarrohemorrágico-ulcerativa difusa aguda y edema submucoso, y severa enterocolitis mucohemorrágica difusa aguda. 

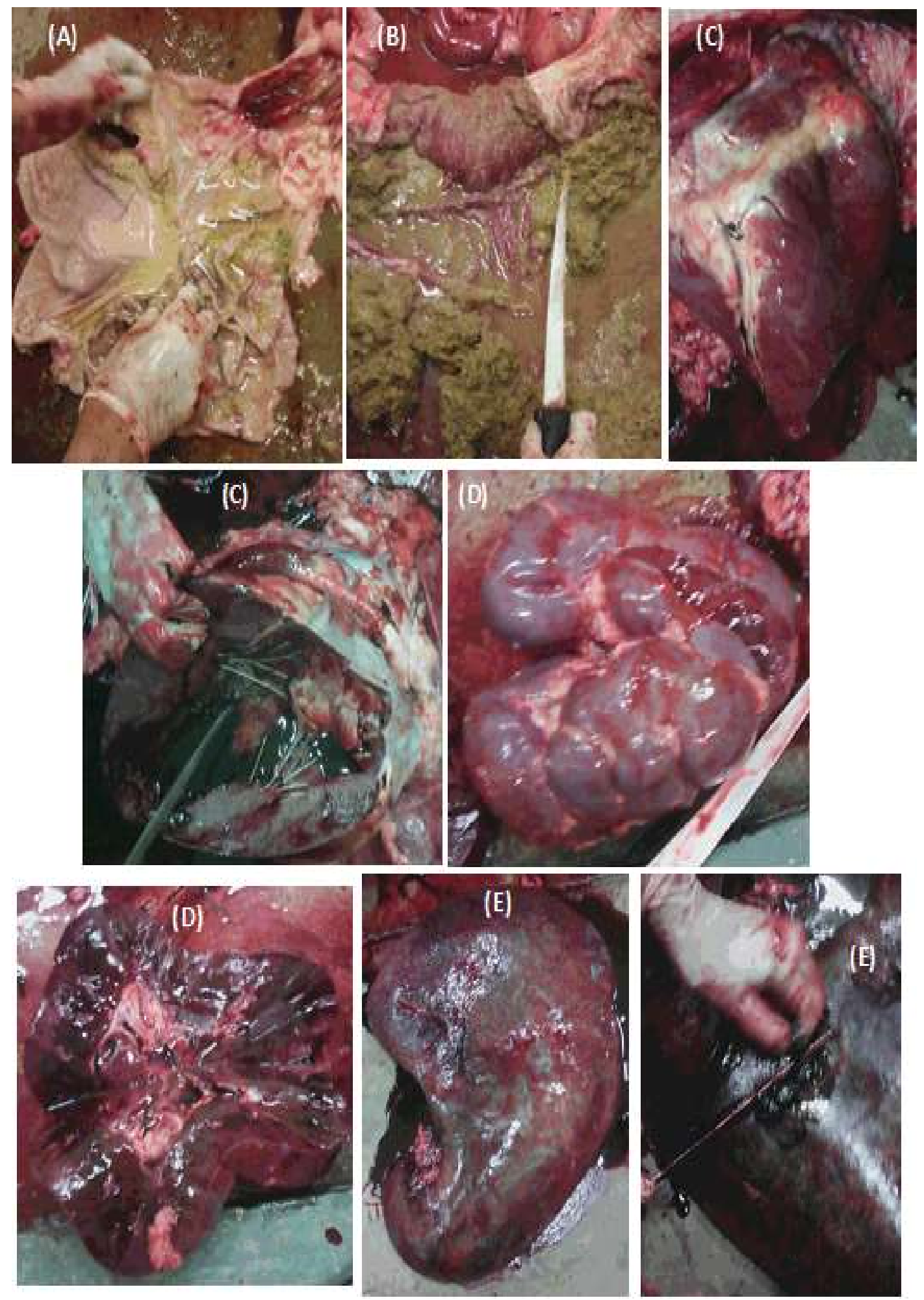

Figura 1. Hallazgos macroscópicos en órganos de una potranca de dos años tratatada con AINES y que murió durante una artroscopia. (A) Gastritis catarro-hemorrágicoulcerativa difusa aguda; (B) Enterocolitis hemorrágica difusa aguda; (C) Ventrículo derecho contraído y con abundante líquido hemorrágico; (D) Porción medular del riñón derecho enrojecida; (E) Esplenomegalia con severa congestión esplénica 

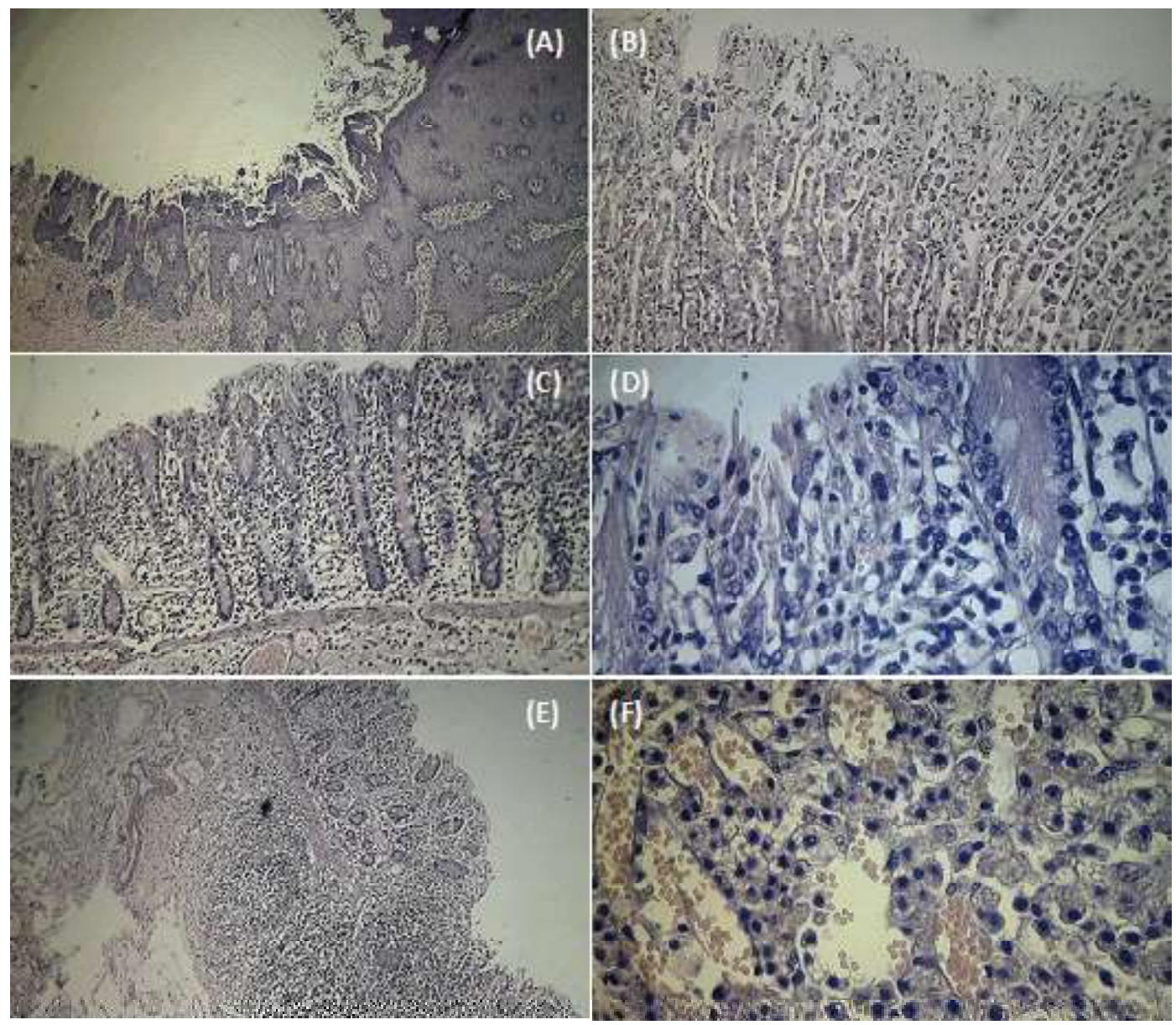

Figura 2. Hallazgos microscópicos en órganos de una potranca de dos años tratatada con AINES y que murió durante una artroscopia. (A) Estómago con marcada exfoliación del epitelio; (B) Gastritis catarral mucosa severa y descamación del epitelio glandular; (C) Colitis no supurativa con descamación, infiltración de células inflamatorias; (D) Desprendimiento del epitelio de la mucosa del intestino grueso; (E) Ganglios mesentéricos con folículos linfoides en marcada hiperplasia con edema inter y extrafolicular; (F) Congestión y focos de hemorragia en la glándula adrenal

\section{Hallazgos Histopatológicos (ver Figura 2)}

- La corteza renal con las células epitelio tubulares proximales y asa de Henle hinchadas por tumefacción turbia que en algunas zonas obliteran sus lúmenes. Glomérulos dilatados con capilares severamente congestionados. La médula con túbulos mostrando cambios similares a los corticales.
- Bazo con la pulpa roja severamente aumentada de volumen. La pulpa blanca con severa depleción linfoide.

- La región glandular del estómago con mucosa en marcada exfoliación de su epitelio y necrosis. La submucosa infiltrada por abundantes linfocitos y escaso tejido conectivo fibroso. La región no glandular con foco de necrosis epitelial superficial y exfoliación con trombosis. 
- Intestino delgado con severa denudación del epitelio de revestimiento de las vellosidades y necrosis con leve hiperplasia de las glándulas de Lieber-kühn y folículos linfoides de placas de Peyer con congestión y trombosis.

- En el intestino grueso se observa severa denudación del epitelio de revestimiento, hiperplasia de los folículos linfoides erráticos submucosos, congestión y trombosis.

- Ganglios mesentéricos con folículos linfoides en marcada hiperplasia, con edema inter y extrafolicular con severa hemorragia cortico medular y trombosis.

- En la adrenal, las células de la corteza y médula se encuentran hinchadas, en degeneración turbia y vacuolar, congestión y focos de hemorragia.

- La musculatura cardiaca sin cambios considerables. Algunas células de Purkinje en degeneración turbia y necrosis.

\section{Diagnósticos Histopatológicos}

Moderada nefrosis tubular aguda, severa esplenomegalia congestiva aguda y severa depleción linfoide, severa gastritis necrótica no supurativa ulcerativa subaguda, severa enterocolitis necrotizante difusa aguda, severa linfoadenitis hemorrágica difusa aguda, trombosis con depleción linfoide y edema, moderada adrenopatosis difusa aguda y congestión y leve necrosis de células de Purkinje miocárdica.

\section{Causa de Muerte}

Paro cardio-respiratorio y coagulopatía con disturbio circulatorio y proceso ulcerogénico gástrico asociado a shock iatrogénico por fenilbutazona.

\section{Discusión}

La cirugía para la corrección de la lesión carpiana se realizó sin mayores evaluaciones previas, dada la corta edad del animal y la ausencia de antecedentes alarmantes que comprometieran su salud. No obstante, es probable que la potranca ingresara a la cirugía con baja frecuencia cardiaca que pudo haber sido promovida por la severa lesión gastrointestinal, haciendo que el animal no estuviera en condiciones favorables para una cirugía.

Los fármacos de inducción y mantenimiento quirúrgico como la xilacina, al ser un agonista cardiaco y vascular $\alpha_{2}$-adrenérgico pueden predisponer a la baja de frecuencia cardiaca (bradicardia sinusal) e hipertensión inicial seguida de una más duradera y bloqueos atrioventriculares (Sumano y Ocampo, 2006a), que junto al promazil (maleato de acepromacina) y sevorane (sevoflurano), exacerbaron aún más la hipotensión conduciendo al daño nervioso de las células de Purkinje miocárdicas, condicionando al paro cardiaco y, finalmente, la muerte del animal (Sumano y Ocampo, 2006a).

El daño hemorrágico y ulcerativo del tracto gastrointestinal (siendo mayor en el estómago) también promueve la hipotensión, las cuales se asocian por el uso de la fenilbutazona al inhibir la ciclooxigenasa $1 \mathrm{y}$ suprimir la producción de prostaglandinas, causando menos producción de moco. Este mantiene la integridad de la mucosa gástrica e intestinal, además de incrementar la vía lipooxigenasa aumentando los ácidos hidroxiperoxi-eicosatetranoicos, leucotrienos y radicales libres, siendo estas sustancias más nocivas al estómago (Peskar et al., 2001). Si se le adiciona el constante estrés por las rutinas que a diario recibe el animal, será mayor el riesgo en animales jóvenes.

Por otro lado, el animal presentó una severa enterocolitis hemorrágica aguda difusa, lo que produce infiltración de células inflamatorias y un aumento de la permeabilidad capilar a nivel de la mucosa y submucosa intestinal, que conduce a edema por pérdida de proteínas a través de la luz intestinal y a una reducción considerable de la presión oncótica del plasma (Dowling, 2002). La leve linfadenitis hemorrágica de los linfonódulos mesentéricos podría deberse a la acción de 
los AINEs, ya que es conocido que estos causan disrupción de la barrera epitelial, lo que resulta en un incremento de la permeabilidad a las toxinas bacterianas (Magdesian, 2003).

El uso de los AINEs también compromete la función renal causando nefropatías al inhibir la síntesis de las prostaglandinas vasopresoras que regulan el flujo sanguíneo renal, la filtración glomerular, el transporte tubular de iones, la liberación de renina y el metabolismo de agua. A pesar de ello, no se evidenciaron lesiones renales severas a nivel macroscópico, aunque sí a nivel microscópico, ya que se menciona que esto se manifiesta más en animales deshidratados (Monreal et al., 2004), donde en este caso no se encontraron indicios de deshidratación.

Es probable que el uso de la fenilbutazona haya influido indirectamente el problema de la coagulación, ya que este inhibe la agregación plaquetaria (Bricks et al., 2005); sin embargo, en equinos este problema es poco frecuente, aunque resultó extraño porque el animal no había presentado problemas de tipo hemorrágico, el cual solo se evidenció durante la necropsia. Es probable que también haya habido mielotoxicosis, causado depresión de los valores de sangre.

Es muy frecuente el uso indiscriminado de los AINEs, en especial de la fenilbutazona en el tratamiento de lesiones en equinos; sin embargo, usualmente se utilizan dosis altas y prolongadas de este fármaco, lo que predispone a desarrollar diversos cuadros de toxicidad en caballos (Jones, 2003; Melo et al., 2009). MacKay et al. (1983) señalan que la dosis endovenosa no debe exceder de $2 \mathrm{~g} /$ día por 5 días, continuando con una administración oral en caso sea necesario. La potranca, según mencionó el Médico Veterinario, había estado recibiendo fenilbutazona, vía endovenosa, por nueve días seguidos, lo que produjo un cuadro de toxicosis evidenciado por las lesiones anatomopatológicas diagnosticadas en la necropsia.

\section{Literatura Citada}

1. Botting RM. 2006. Cyclooxygenase: past, present and future. A tribute to John R. Vane (1927-2004). J Therm Biol 31: 208-219. doi: 10.1016/j.jtherbio.2005.11.008

2. Bricks LF, Silva CAA. 2005. Toxicidade dos anti-inflamatórios nãohormonais. Pediatria 27: 181-193.

3. Dowling P. 2002. Adverse drug reactions in horses. Clin Tech Equine Pract 1: 58-67. doi: 10.1053/ctep.2002.34868

4. Halper J, Kim B, Khan A, Yoon J, Mueller P. 2006. Degenerative suspensory ligament desmitis as a systemic disorder characterized by proteoglycan accumulation. BMC Vet Res 2: 12. doi: 10.1186/1746-6148-2-12

5. Jones $S$. 2003. Right dorsal colitis. In: Robinson N (ed). Current therapy in equine medicine. $5^{\text {th }}$ ed. Philadelphia, USA. Saunders. p 141-143.

6. Keegan KG, Messer NT, Reed SK, Wilson DA, Kramer J. 2008. Effectiveness of administration of phenylbutazone alone or concurrent administration of phenylbutazone and flunixin meglumine to alleviate lameness in horses. Am J Vet Res 69: 167-173. doi: 10.2460/ajvr.69.2.167

7. Kollias-Baker C, Cox K. 2004. Nonsteroidal anti-inflammatory drugs. In: Bertone JJ, Horspool L (eds). Equine clinical pharmacology. Philadelphia, USA: Saunders. p 247-266.

8. Lees P, Higgins AJ. 1985. Clinical pharmacology and therapeutic uses of non-steroidal anti-inflammatory drugs in the horse. Equine Vet J 17:83-96. doi: 10.1111/j.2042-3306.1985.tb02056.x

9. Lees P, Higgins AJ. 1987. Physiological, biochemical and haematological effects on horses of a phenylbutazone paste. Vet Rec 121: 56-60. doi: 10.1136/ vr.121.3.56 
10. MacAllister CG. 1994. Nonsteroidal anti-inflammatory drugs: their mechanism of action and clinical uses in horses. Vet Med 89: 237-240.

11. MacKay RJ, French TW, Nguyen HT, Mayhew IG. 1983. Effects of large doses of phenylbutazone administration to horses. Am J Vet Res 44: 774-780.

12. Magdesian K. 2003. Clostridium difficile infection. In: Robinson N (ed). Current therapy in equine medicine. $5^{\text {th }}$ ed. Philadelphia, USA: Saunders. p 166-169.

13. Martín-Jiménez T, MG Papich. 2002. Prostaglandinas y antiinflamatorios no esteroideos. En: Botana LM, Landoni F, Martín-Jiménez T (eds). Farmacología y terapéutica veterinaria. Madrid, España: McGraw-Hill. p 350-374.

14. Matthews N, Carrol G 2007. Review of equine analgesics and pain management. Proc $53^{\text {rd }}$ Annual Convention of the AAEP. Orlando, USA.

15. Mealey KL, Matthews NS, Peck KE, Ray AC, Taylor TS. 1997. Comparative pharmacokinetics of phenylbutazone and its metabolite oxyphenbutazone in clinically normal horses and donkeys. Am J Vet Res 58: 53-55.

16. Melo UP, Fiório R, Souza A, Ferreira $C$, Mocaiber S. 2009. Intoxicação por fenilbutazona em equino: relato de caso Acta Vet Brasilica 3: 111-116. doi: 10.21708/avb.2009.3.2.1265

17. Monreal D, Sabat D, Segura I, Mayós I, Homedes J. 2004. Lower gastric ulcerogenic effect of suxibuzone compared to phenylbutazone when administered orally to horses. Res Vet Sci 76:145-149.

18. Peskar BM, Maricic N, Gretzera B, Schuligoi R, Schmassmann A. 2001. Role of cyclooxygenase-2 in gastric mucosal defense. Life Sci 69: 2993-3003.

19. Skarra TK. 1994. Forgiftning med fynylbutazon hos hest. Norsk Vet Tidsskr 106: 443-449.

20. Sumano LH, Ocampo CL. $2006 a$. Tranquilizantes. En: Farmacología veterinaria, $3^{\mathrm{a}}$ ed. México: McGraw Hill Interamericana. p 709-730.

21. Sumano LH, Ocampo CL. 2006 . Analgésicos no narcóticos. Farmacología veterinaria, $3^{\mathrm{a}}$ ed. México: McGraw Hill Interamericana. p 780-807.

22. Tobin T, Chay S, Kamerling S, Woods WE, Weckman TJ, Blake JW, Lees P. 1986. Phenylbutazone in the horse: a review. J Vet Pharmacol Ther 9: 1-25. doi: 10.1111/j.1365-2885.1986.tb00008.x 